\title{
Evaluation of Water Distribution Jointed Pipe Networks under Transient Ground Motions
}

\author{
Alireza Boorboor, Mahmood Hosseini \\ Department of Structure, International Institute of Earthquake Engineering and Seismology, Tehran, Iran \\ Email: a.boorboor@iiees.ac.ir, Hosseini@iiees.ac.ir
}

Received 20 March 2015; accepted 19 May 2015; published 22 May 2015

Copyright (C) 2015 by authors and Scientific Research Publishing Inc.

This work is licensed under the Creative Commons Attribution International License (CC BY). http://creativecommons.org/licenses/by/4.0/

c) (i) Open Access

\section{Abstract}

In this study, a new method is proposed to evaluate the seismic behavior of buried jointed water pipeline networks subjected to wave propagation. First, using finite element method and solid elements, different kinds of currently used connections in the network are modeled, and their nonlinear behavior in all directions is obtained. Second, a 950-meter long network consisting of ductile iron pipes segments of 6-meter length and springs characterizing the connections, are modeled using beam elements. Three-component displacement record of the Tabas earthquake is applied to the network considering the time lag between support inputs, and the nonlinear soilpipe interaction. The record is applied once in North-South direction and once in East-West direction with different wave propagation velocities. Results of interest such as stress values and rotations at various points of the network are then obtained, and critical points are introduced in each direction. Results show that the points other than the critical ones at the network intersections remain elastic.

\section{Keywords}

Finite Element Method, Ductile Iron Connections, Three Component Displacement Records, Nonlinear Soil-Pipe Interaction

\section{Introduction}

Water supply networks are one of the main components of urban infrastructures and their continuous and uninterrupted operation is of great importance in today's life. These networks are vulnerable to seismic wave propagation, and adverse consequences followed directly or indirectly from damages, affect the citizens. Since the 
jointed pipe network constitutes a significant part of Tehran's water supply system, investigating the network operation during the design earthquake and ensuring its functionality after the earthquake is necessary.

For the first time in 1930, earthquake effects on water networks were considered. However, analytical and numerical studies have started since two decades ago [1]. So far, seismic damage assessment on water distribution networks has been conducted by using various methods including theoretical methods such as artificial neural network [2]. Due to difficulties in describing ground-motion intensity over a region and since the link between the ground-motion intensities and lifeline performance is usually not available in closed form, Jayaram and Baker [3] proposed a simulation-based framework for developing a small but stochastically-representative catalog of earthquake ground-motion intensity maps that can be used for lifeline risk assessment. In references [4] and [5] reports on the earthquake damage on jointed pipe networks are issued. Toprak et al. [6] evaluated the performance of the water supply system in Denizli, Turkey. They compared the relative effects of transient ground deformations and permanent ground deformations based on maps of liquefiable soil and zones of predicted lateral ground displacements.

During the past earthquakes, most of the jointed pipe network failures have occurred at connections, where connection pull-out, cut and crushing (concrete pipe connections) have been reported [1]. A large number of the studies have been conducted on continuous pipeline networks, and because of the complexities in jointed pipe networks compared to the continuous ones, fewer studies on jointed pipe networks considering connection behavior have been made. Most of the studies on jointed pipe networks have been based on observations or estimations, and only in the past two decades, thanks to software and laboratory advances, research in this area has been expanded. Abdoun et al. [7] conducted an experimental study on the behavior of buried continuous pipeline subjected to ground faulting. Karamitros et al. [8] presented a rigorous method to calculate the response of continuous pipes considering the axial and bending stiffness. Junhee Kim et al. [9] carried out the experimentation of a reinforced concrete segmented concrete pipeline. Accurate measures of pipeline displacements and strains were captured up to the compressive and flexural failure of the pipeline joints.

The present study is carried out on a part of water supply network of Tehran metropolis in which ductile iron pipes of 6 meter length and connections of Tyton and Bolted gland types are used. Focusing on non-linear behavior of the connections, the present study tries to identify mechanically vulnerable points of the network. The analyses are performed using appropriate Finite Element (FE) software. Details of the study are presented in the following sections.

\section{Introducing the Studied Network and Modeling of the Network Connections}

Figure 1 shows the sketch of the studied network, and Table 1 shows the pipeline lengths and the connection types in the network.

In this section, the connections are modeled numerically, and their stiffness components are obtained by displacement loading in different directions. Table 2 shows the mechanical properties of ductile iron pipes and the gaskets.

Table 1. Length of the pipes and types of the connections in the network.

\begin{tabular}{lc}
\hline 1 & Tyton 150 pipeline $\mathrm{L}=251 \mathrm{~m}$ \\
2 & Tyton 150 pipeline $\mathrm{L}=96 \mathrm{~m}$ \\
3 & Tyton 200 pipeline $\mathrm{L}=290 \mathrm{~m}$ \\
4 & Tyton 200 pipeline $\mathrm{L}=36 \mathrm{~m}$ \\
5 & Tyton 300 pipeline $\mathrm{L}=234 \mathrm{~m}$ \\
6 & Tyton 200 pipeline $\mathrm{L}=42 \mathrm{~m}$ \\
A & Bend 200, connected to the pipeline from both ends \\
B & $200-300$ culvert, connected to the pipeline from one end (mono) \\
C & Bend 300, connected to the pipeline from one end \\
D & 200 to 300 culvert, connected to the pipeline from both ends (double) \\
\hline
\end{tabular}




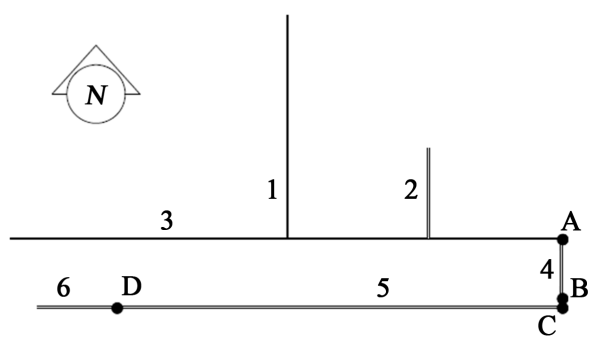

Figure 1. The sketch of the studied network.

Table 2. Mechanical properties of the materials.

\begin{tabular}{ccc}
\hline Property & Ductile iron & Gaskets \\
\hline$\left(\mathrm{kg} / \mathrm{m}^{3}\right)$ & 7050 & 1900 \\
$\mathrm{E}\left(\mathrm{N} / \mathrm{m}^{2}\right)$ & $1.70 \mathrm{E}+11$ & $2.77 \mathrm{E}+06$ \\
$\mathrm{~V}$ & 0.275 & 0.497 \\
$\sigma_{\mathrm{y}}\left(\mathrm{N} / \mathrm{m}^{2}\right)$ & $3.00 \mathrm{E}+08$ & $6.00 \mathrm{E}+06$ \\
$\sigma_{\mathrm{u}}\left(\mathrm{N} / \mathrm{m}^{2}\right)$ & $4.2 \mathrm{e} 8$ & $6.50 \mathrm{E}+06$ \\
$\varepsilon \mathrm{u}$ & 0.1 & 3 \\
\hline
\end{tabular}

The material behavior is considered as bi-linear. For considering pipe-gasket and pipe-pipe interaction, two normal and tangent components are considered as follows:

- For the normal component, the two individual segments cannot pass through each other, and the two interacting surfaces can be separated.

- For the tangent component, an appropriate friction coefficient is defined.

Before conducting the analyses on the pipe connections, verification of the FE program is necessary. For this purpose, the numerical modeling solutions are verified by the experimental results. The maximum error among the verification cases is less than $11 \%$, and the main reason behind this error is the lack of accurate modeling for the material behavior as a result of insufficient information available.

\subsection{Tyton Connections}

In Tyton Connections, the gap between spigot and socket due to the manufacturing tolerances, and operational limits, has a significant impact on the results and by increasing the gap, the flexural behavior of the connection becomes softer. In the productions of the company, this manufacturing tolerance is equal to $1 \mathrm{~mm}$ and this value is used in modeling. In Figure 2, stress contours, deformation and nonlinear behavior of Tyton connections under compressive loading are shown. It should be mentioned that, the red color represents areas of the maximum stress, while the blue shows areas of the least stress.

As can be seen in Figure 2(b), there is a $4 \mathrm{~mm}$ distance (gap) between spigot and the socket in axial direction. The only resistant factor against spigot inward movement is its friction with the gasket that can be ignored. When the gap is finished, two cast iron segments (spigot and socket) start to contact each other, and from this stage on, the inward movement is accompanied by a high axial stiffness until the stress at the edge of the plain head (spigot) reaches the yield point. This yielding occurs in the entire pipe section, simultaneously. Finally, the stress reaches its ultimate value. Since the only resisting factor against spigot's movement in tension is its friction with the gasket, for Tyton connections, a small value is considered as the tensile stiffness. Figure 3 shows the stress contours, deformation and behavior of the Tyton connections in shear.

As one can observe in Figure 3, there is a distance between socket and spigot in shear as well as the axial direction that causes a very soft behavior at the beginning of the movement. With the socket and spigot colliding, the behavior is stiffened until the spigot yields in its contact point with the socket, because the thickness of the spigot is less than the socket head. Unlike the compressive mode, in shear mode, the yielding gradually spreads from the contact points of two pipes, and by increasing the contact surface of the two segments, yielding area 


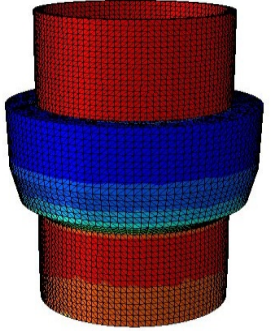

(a)

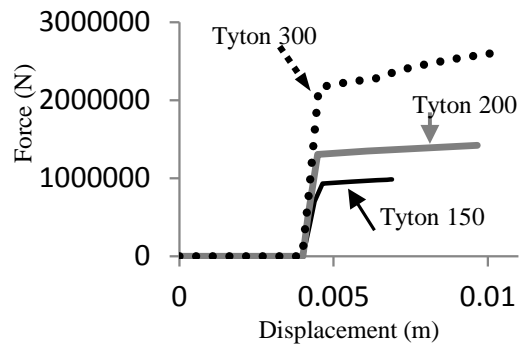

(b)

Figure 2. Tyton connections in compression. (a) Stress contours and compressive deformation; (b) Compressive behavior.

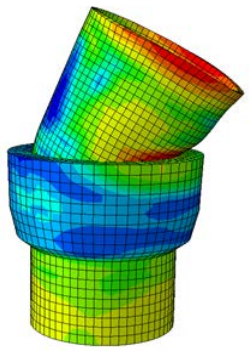

(a)

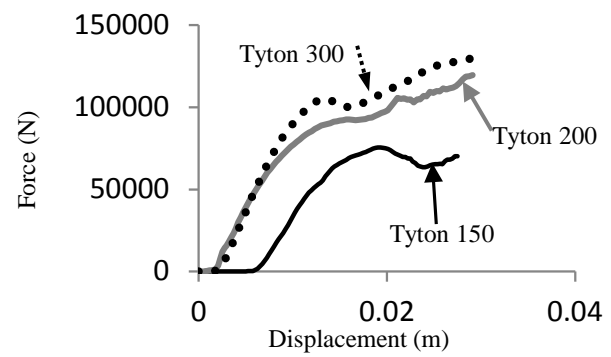

(b)

Figure 3. Tyton connections in shear. (a) Stress contours and shear deformation; (b) Shear behavior.

expands. It is worth mentioning that Tyton connections of different diameters are modeled with different lengths, and the results show that the length parameter does affect the connection behavior. Thus, it can be deduced that the shear and flexural behaviors of Tyton connections (showed in Figure 3) cannot be compared based only on their diameters.

For the flexural loading, the end of the socket is fixed, and by defining a constraint point at the end of the spigot, the whole section is restrained. Afterwards, by rotating the point, the section also rotates uniformly. To determine the effect of the gap between the socket and spigot, the flexural behavior of Tyton 150 for three different gaps of 0,1 and $3 \mathrm{~mm}$ is evaluated and the results of $1 \mathrm{~mm}$ gap are used in the network. In Figure 4, stress contours, deformation and flexural behavior of Tyton connections are shown. As can be observed in Figure 4(b), $1 \mathrm{~mm}$ gap has no significant effect on the ultimate strength and only causes slackness (initial soft behavior) at the beginning of the flexure. However, by increasing the gap from 1 to $3 \mathrm{~mm}$, a significant increase in the initial slackness and a decrease in the stiffness can be noticed. In addition, the ultimate strength is significantly reduced due to the drop in the effective cross section for bending which, leads to stress concentration.

\subsection{Bolted Gland Connections}

Figures 5-10 show stress contours, deformation and behavior of the 200 - 300 Bolted gland connection in compression and shear loadings, respectively.

According to Figure 5, in the Bolted gland connection (mono), stress concentration occurs near the $200 \mathrm{~mm}$ end, and in $300 \mathrm{~mm}$ end, stresses decrease due to the increase in the cross section. According to Figure 6, it can be stated that when the thickness of the 200 - 300 culvert with two socket heads (bolted gland double) is more than that of the two attached pipes, stress concentration in the two pipes and the culvert segment remains almost within the linear limits.

Considering Figure 9, one can note that since the culvert segment has been attached to the straight pipes at both ends, it can easily rotate. This makes the connection softer and reduces its ultimate shear resistance so that no high stress would be imposed to the culvert. In the connection of the pipes and the culvert, stress values increase in the pipes, while in the culvert segment, stress values remain in the elastic range due to its higher thickness value. 


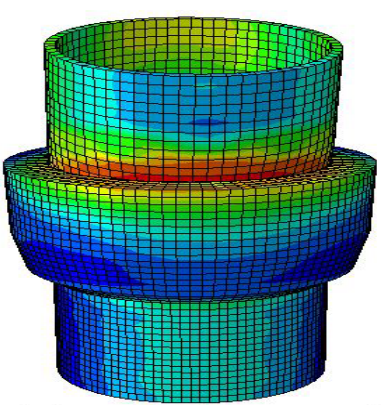

(a)

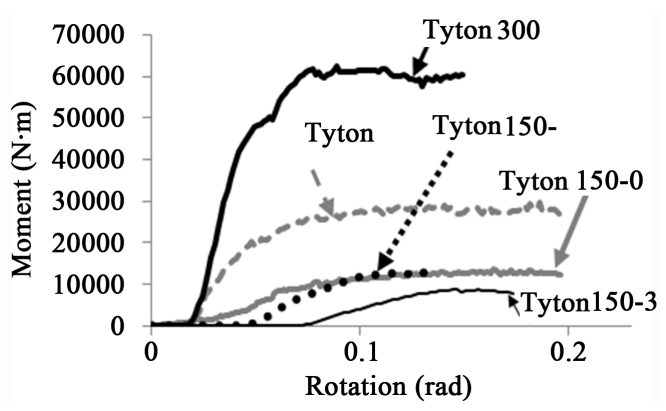

(b)

Figure 4. Tyton connections in bending. (a) Stress contour and flexural deformation; (b) Flexural behavior.

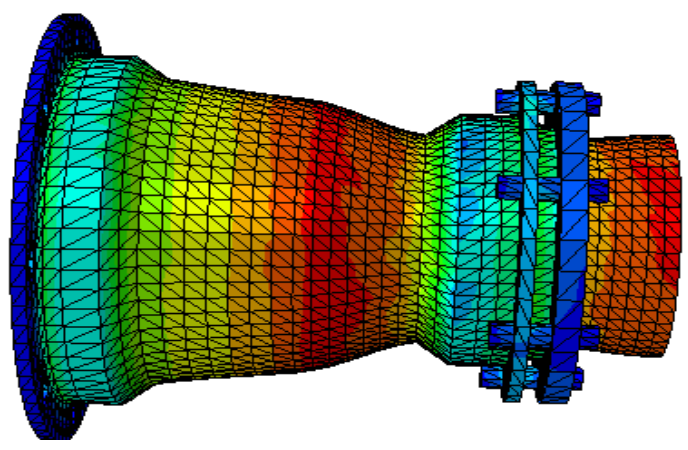

Figure 5. Stress contours and compressive deformation of Bolted gland connection attached to the bend (mono).

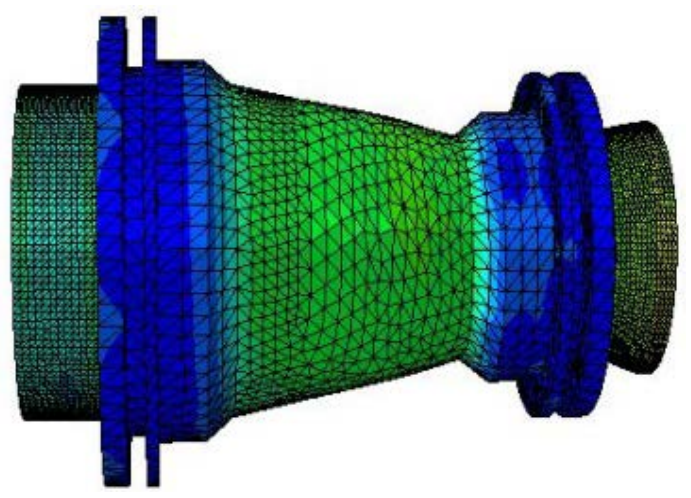

Figure 6. Stress contours and compressive deformation of Bolted gland connection attached to the straight pipe (double).

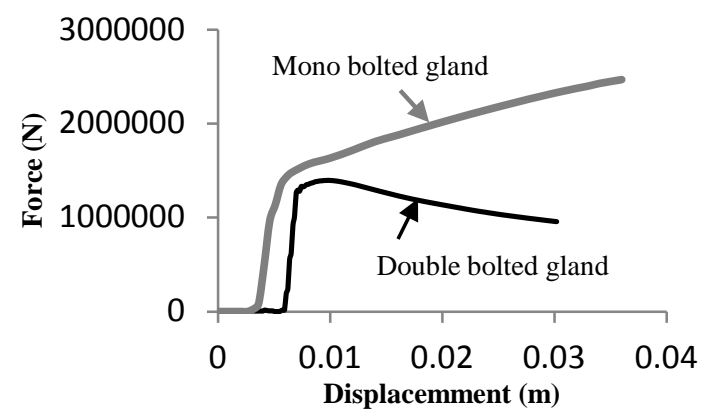

Figure 7. Compressive behavior of Bolted gland connections. 


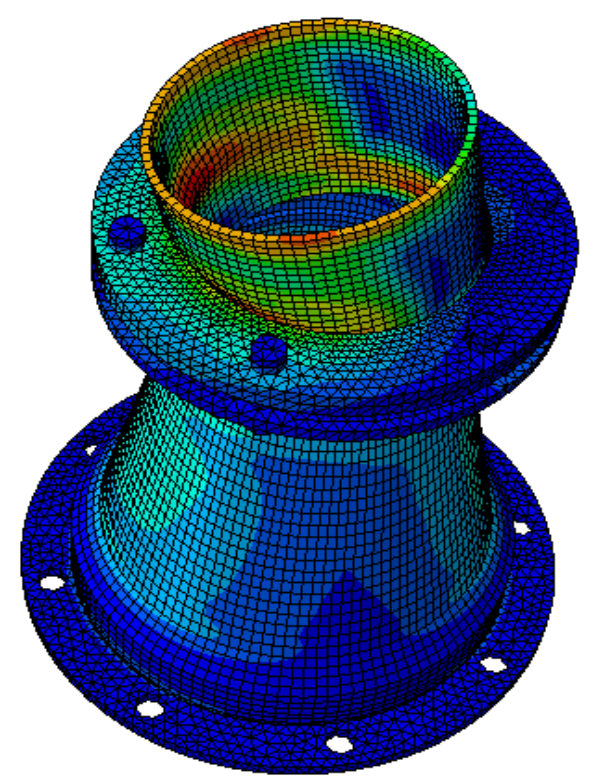

Figure 8. Stress contours and shear deformation of bolted gland connections attached to the bend.

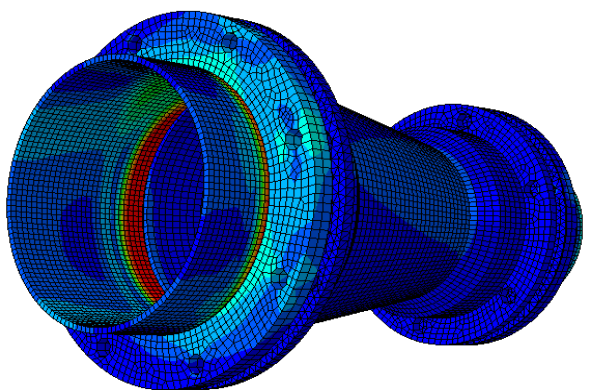

Figure 9. Stress contours and shear deformation of bolted gland connections attached to the straight pipe.

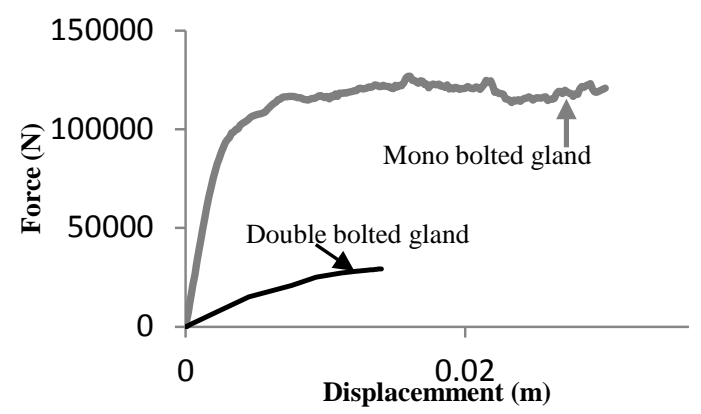

Figure 10. Shear behavior of Bolted gland connections.

\subsection{Bend Connections}

Figures 11-16 show stress contours, deformation, and behavior of the bend connections in compression and shear respectively.

In $200 \mathrm{~mm}$ bend connection, similar to 200 - $300 \mathrm{~mm}$ culvert with two spigots, the connected pipes are weaker in shear direction compared to the bend segment, and stress in the bend remains in elastic range. As can be seen in Figure 11 and Figure 12, stress in the screws can also be neglected. It can be seen from Figure 12 that 


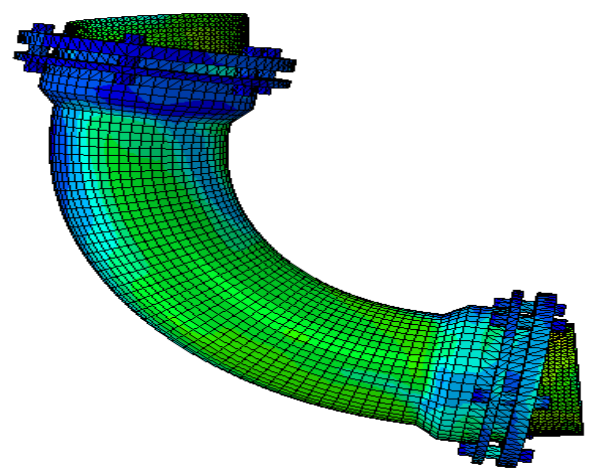

Figure 11. Stress contours and compressive deformation of the $200 \mathrm{~mm}$ bend.

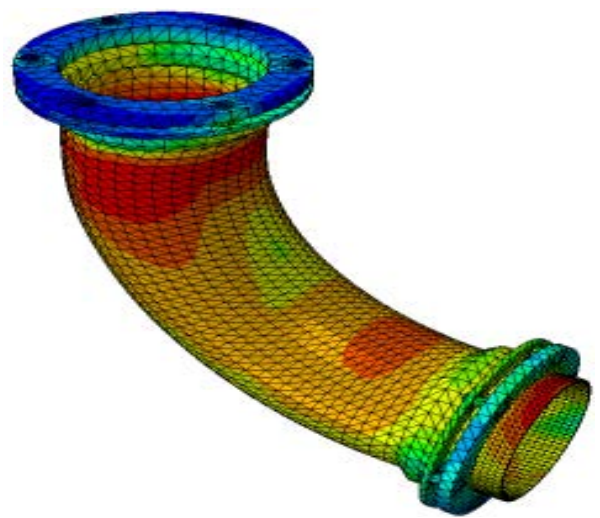

Figure 12. Stress contours and compressive deformation of the $300 \mathrm{~mm}$ bend.

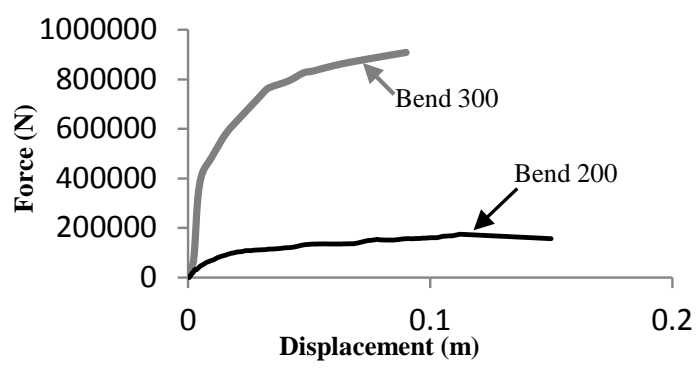

Figure 13. Compressive behavior of bend connections.

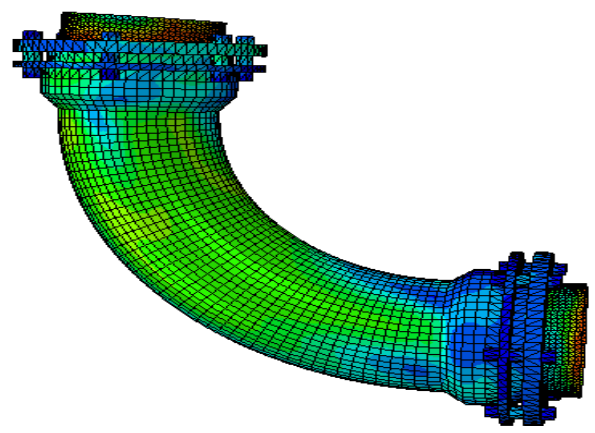

Figure 14. Stress contours and lateral deformation of $200 \mathrm{~mm}$ bend. 


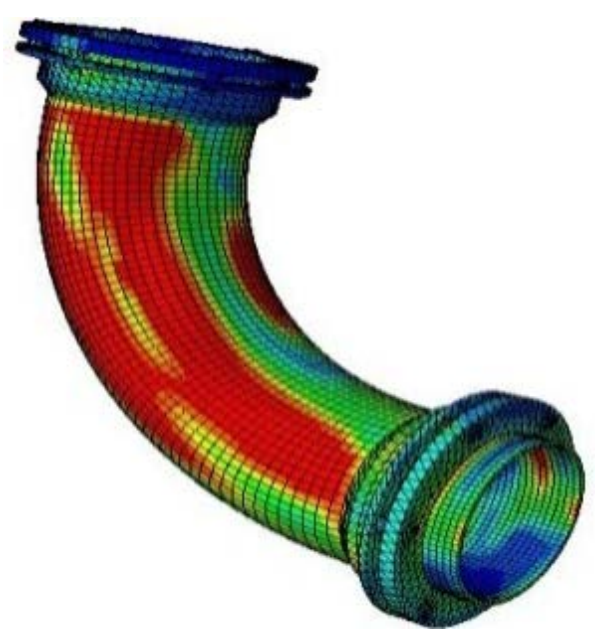

Figure 15. Stress contours and lateral deformation of 300 mm bend.

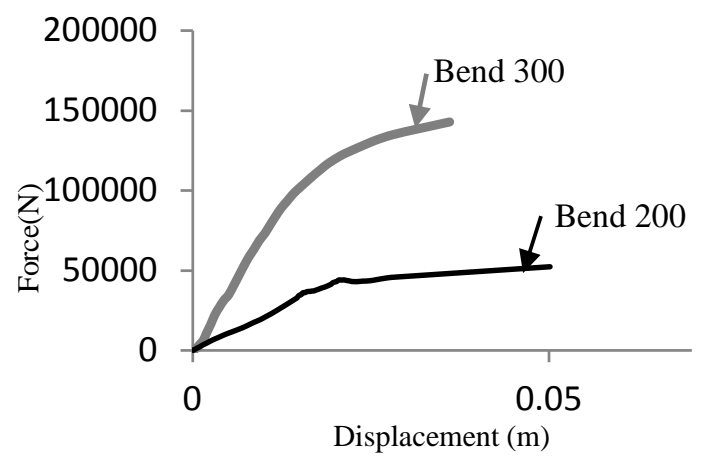

Figure 16. Lateral behavior of the bends.

the $300 \mathrm{~mm}$ bend is attached to 1 spigot. In this connection, unlike the $200 \mathrm{~mm}$ bend, there is a significant stress in the body. In this case, the segment of the bend, in addition to the transmitted force from spigot, should overcome the induced moment. This moment causes plastic stress near the support (in the side with no pipe). In the support itself, because of its considerable thickness, stress remains in elastic range. The behavior of $300 \mathrm{~mm}$ bend is completely different from $200 \mathrm{~mm}$ bend and the capacities of these two bends are not comparable.

By comparing the behavior of the connections graphs, it can be seen that Tyton connections gaps cause softer behavior in comparison to Bolted gland connections. For example, the maximum elastic strength of 200-300 Bolted gland connection with 1 spigot (mono) in shear is about 30\% higher than the corresponding force of 200 mm Tyton. The main fault of the Tyton connections is in tension, as the only resistant factor is the friction between the gasket and the spigot, which is very low and is actually negligible.

Complementary information about the behavior of the current connections is presented in the main report of the work [10].

\section{Modeling and Seismic Analysis of the Network}

In this study, a part of Tehran water distribution pipeline network is considered with an overall length of 950 meters. It is constituted of a number of bends, culverts and straight pipes. The sketch of this network including the types of connections and pipe diameters was shown in Figure 1.

\subsection{Soil-Pipe Interaction}

This interaction has been taken into account based on reference [11], in which the behavior of soil is modeled by bilinear springs, whose specifications depend on the pipe diameter, soil type and density, its internal friction an- 
gle, and the burial depth of pipe. In the modeling, a burial depth of 1.5 meters and compacted sand are considered. Equivalent soil springs are defined with translational properties in three directions, while the equivalent springs of the connections between two pipe segments have three translational and three rotational components. It is necessary to note that according to Figure 17, equivalent soil springs are placed every 1 meter along the pipe, and at both ends of the connection.

For modeling boundary conditions, after testing different scenarios, it is finally decided that the rotations being restrained at four boundary points (ends of the pipelines) in all three directions and the translations being free is the situation most consistent with the actuality, because, the least local stress is caused in the foregoing scenario.

\subsection{The Minimum Effective Length}

The minimum effective length in pipelines was defined by Tahamoli-Hosseini [12]. They determined the term for the continuous pipelines (welded connections that can be supposed as continuous pipes) [12]. In short, the minimum length for which the boundary conditions do not affect the response of the midpoint of the pipeline is called the minimum effective length. To determine this length for jointed pipelines, two pipelines with lengths of 30 and 60 meters and a diameter of $150 \mathrm{~mm}$ are considered. A sinusoidal wave with equation y0 $\sin (2 \pi \mathrm{t} / \mathrm{T})$, (shown in Figure 18) has been applied to the spring supports with a phase difference, which depends on the shear wave velocity in soil. T (period of the wave) equals $1 \mathrm{sec}$, and Vs (the shear velocity) equals $150 \mathrm{~m} / \mathrm{s}$.

According to Figure 19, it can be concluded that in jointed pipelines, the boundary conditions have almost no effect on the behavior of the pipelines, even in case of small total lengths as $30 \mathrm{~m}$. It can be deduced that, the

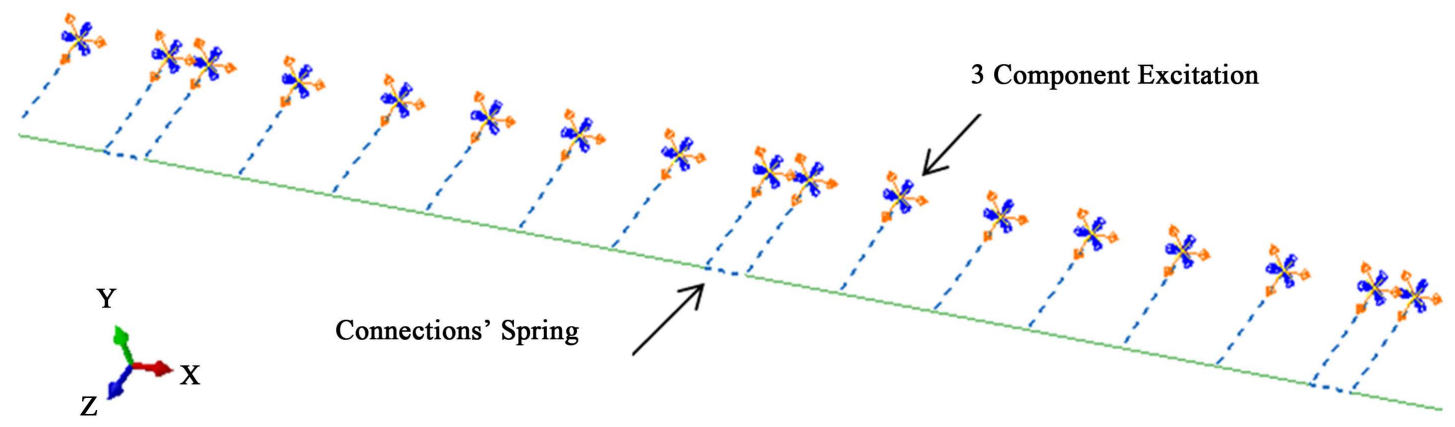

Figure 17. Modeling the soil-pipe interaction and connections of the network.

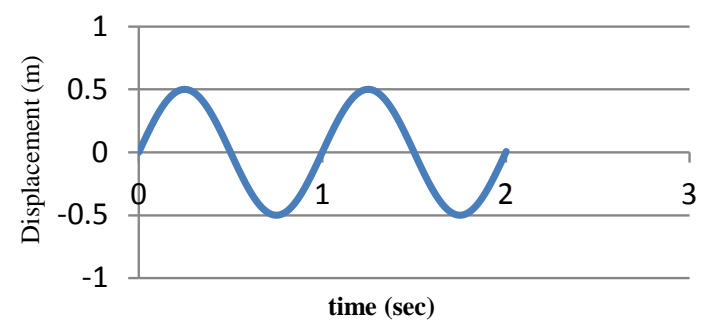

Figure 18. Wave applied to the pipeline to determine the minimum effective length.

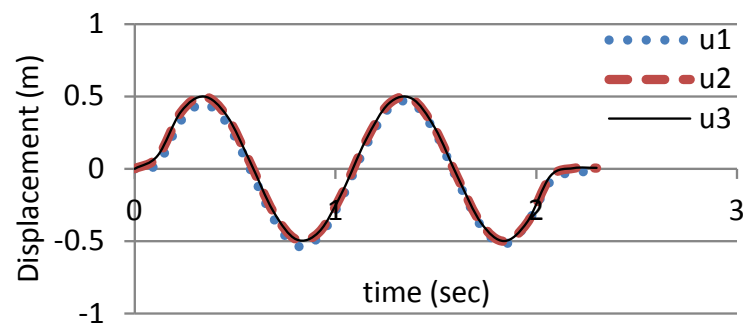

Figure 19. Displacement of the midpoint of the 30 meter long pipeline. 
pipeline is much softer than the dense sand soil and bears almost the whole displacements, while displacements of soil springs are very small.

\subsection{Network Analysis with Tabas Earthquake Record}

Figure 20 shows a schematic sketch of the modeled network with three dimensional loadings. Tabas earthquake record is applied to the spring support with a phase difference, which depends on shear wave velocity in soil. This record is applied once in North-South and once in East-West directions. Figure 21 shows the three component displacement record of the Tabas earthquake.

From the analysis, it is found that, for lines perpendicular to the wave direction, rotations and stresses are very small and lines parallel to the wave direction sustain the maximum damage. In addition, for the critical points of intersections of the branches that are parallel to the wave direction, a high damage is expected.

During the wave movement along North-South direction, the intersection of $150 \mathrm{~mm}$ Tyton Pipeline and 200 $\mathrm{mm}$ Tyton Pipeline is the critical point. That can be as a result of changes in displacement transmission so that the pipeline parallel to wave direction is suddenly cut and the new pipeline becomes perpendicular to the wave direction and the previous pipeline.

Figure 22 shows deformation and stress distribution at the critical point, i.e. the intersection of Tyton 150 pipelines (parallel to the wave direction) and Tyton 200 pipelines (perpendicular to the direction of wave propagation). Wave moves in the N-S direction along Tyton150. In the desired point, the N-S line is cut, and a perpendicular new line is created. This leads to a disturbance in the imposed displacement transmission system, and stress and rotation rise locally. The connection is of Tyton type; therefore, orthogonal pipes can rotate freely around the connection and stress values rise along the pipe.

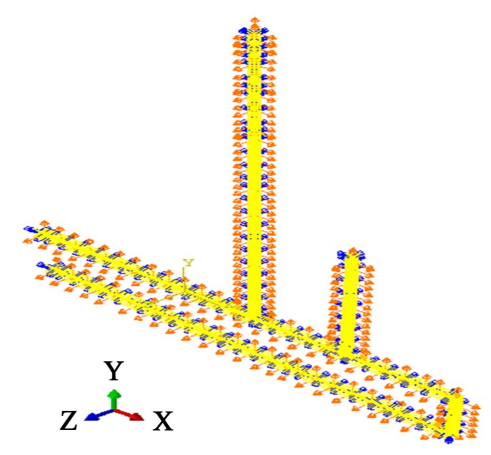

Figure 20. Modeled Network.

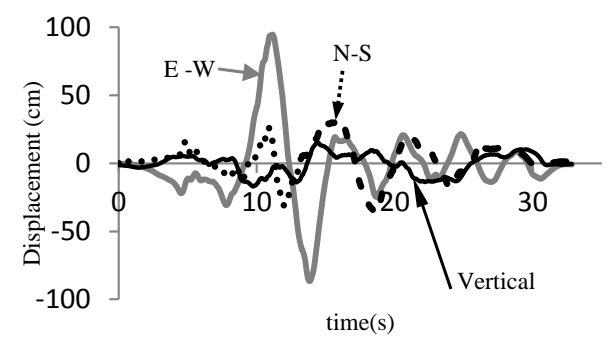

Figure 21. Three component displacement record of the Tabas earthquake.

Figure 22. Deformation of the critical point in N-S direction. 
It is observed from the deformation illustrated in Figure 22 that when the network moves along the N-S direction, the resistant factor against the pipeline of the Tyton 150 is the axial friction of the soil. This factor against perpendicular line is the lateral horizontal component of the soil spring that is much larger than the axial component. It means that the pipeline of Tyton 150, in the axial direction, has a larger freedom of movement than Tyton 200 pipeline; therefore, the pipeline of Tyton 200 would be made to deform as shown in Figure 22.

Similarly, when the wave moves along the East-West direction and is parallel to Tyton 200, Tyton 300 and perpendicular to Tyton 150 pipelines, critical points are the connection points of the bends. Bend connections are of Bolted gland type that are much more resistant against deformation in comparison to Tyton connections. Despite the high level of stress values in Bolted gland connections, their rotations are less than those of Tyton connections. Figure 23 shows the critical points along the E-W direction.

In the following, the results of the analysis are expressed as maximum values of stress and rotation.

\subsubsection{Wave Propagation in North-South Direction (Parallel to Tyton 150 Pipeline)}

Table 3 shows the maximum stress and rotation values of the critical point in the network and also the maximum rotation along Tyton 150 pipeline (away from the ends of the pipeline) for different wave velocities.

Considering the values of stress and rotations in Table 3 and also behavior diagrams of the connections, it can be suggested that only at the critical point, the rotation of the connection exceeds the elastic limit and experiences a non-linear behavior. Moreover, the stress level drops as the velocity increases. This is due to the increase in distance traveled per unit time, thus decreasing the relative displacement (the main cause of the failure) between two desired points. In other words, with increasing the wave velocity, relative displacement between two points with a fixed distance is reduced. The stress in other points including Tyton 300 and Tyton 200 pipelines (except for the critical point) is very small and negligible.

With an increase in the velocity from 150 to 200 meters per second, the stress value (in plastic range) decreases by $2 \%$. And with increasing from 200 to 300 meters per second, the stress value (in elastic range and plastic limit) decreases by $12 \%$.

Figure 24 shows the rotation time history for the both ends of the connection at the intersection of Tyton 150 and Tyton 200 branches (shown in Figure 22), which is the critical point in N-S direction.

\subsubsection{Wave Propagation in East-West Direction (Parallel to the Tyton 200 and Tyton 300 Pipelines)}

Table 4 presents the analysis results in East-West direction. Rotations along Tyton 150 pipeline are very small and negligible, and the stress in the intersection point with Tyton 200 pipeline reaches its maximum value. While the maximum rotation of Tyton 200 and 300 pipelines occurs along the length; hence, it is not rational to

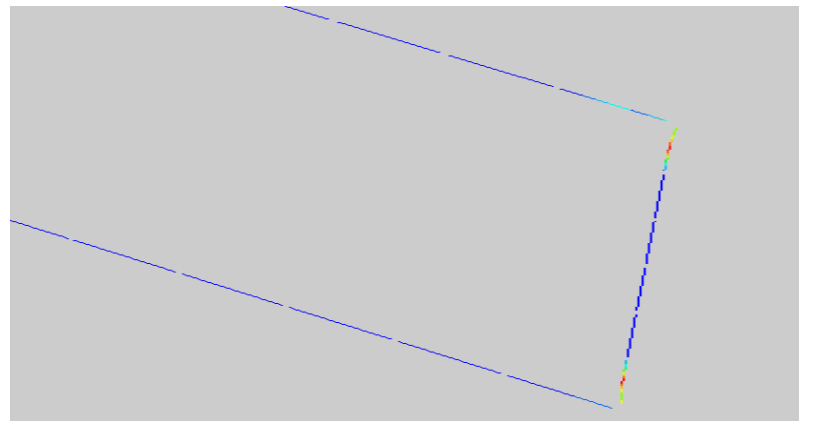

Figure 23. Deformation of the critical point along E-W direction.

Table 3. Results of the analysis with Tabas record along N-S direction.

\begin{tabular}{cccc}
\hline $\mathrm{V}_{\mathrm{S}}(\mathrm{m} / \mathrm{s})$ & 150 & 200 & 300 \\
\hline Stress $\left(\mathrm{N} / \mathrm{m}^{2}\right)$ & $3.08 \mathrm{E}+08$ & $3.01 \mathrm{E}+08$ & $2.69 \mathrm{E}+08$ \\
Tyton150 $(\mathrm{rad})$ & 0.0135 & $1.19 \mathrm{E}-02$ & 0.008 \\
Cr point $(\mathrm{rad})$ & 0.115 & 0.082 & 0.03 \\
\hline
\end{tabular}




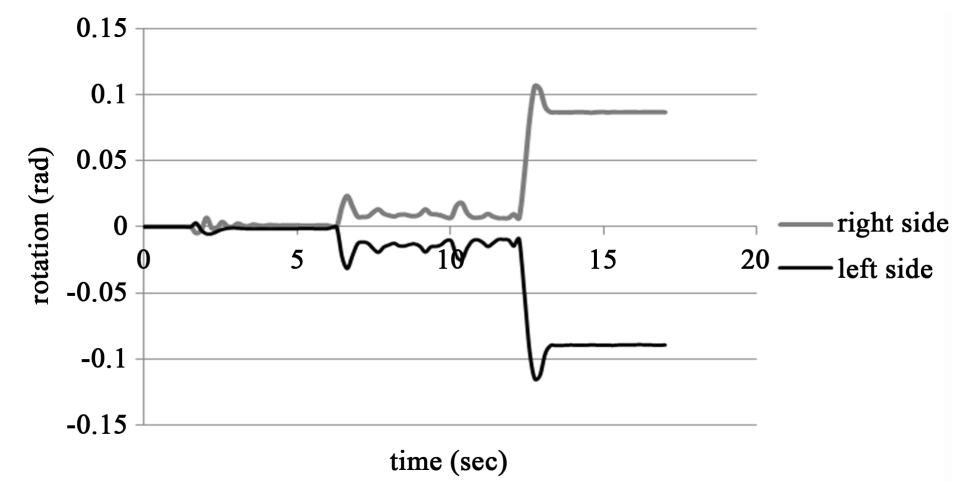

Figure 24. Rotation time history for the both sides of the connection.

Table 4. Results of the analysis with Tabas record along E-W direction.

\begin{tabular}{cccc}
\hline $\mathrm{V}_{\mathrm{S}}(\mathrm{m} / \mathrm{s})$ & 150 & 200 & 300 \\
\hline Stress $\left(\mathrm{N} / \mathrm{m}^{2}\right)$ & $3.20 \mathrm{E}+08$ & $3.10 \mathrm{E}+08$ & $3.00 \mathrm{E}+08$ \\
Tyton $200(\mathrm{rad})$ & 0.00975 & $8.40 \mathrm{E}-03$ & negligible \\
Tyton $300(\mathrm{rad})$ & 0.00825 & 0.0068 & negligible \\
Tyton150 (rad) & 0.04 & 0.024 & $1.18 \mathrm{E}-02$ \\
Bend 200 (rad) & 0.09 & 0.08 & 0.04 \\
Bend 300 (rad) & 0.023 & 0.02 & 0.01 \\
\hline
\end{tabular}

compare the maximum rotation of the three pipelines.

According to Table 4, in East-West case similar to North-South case, stress in connections, exceeds the elastic limit only at the critical points, and the rest of the network remains in the elastic range. Considering the amount of rotation of Tyton 200 and 300 pipelines, it can be observed that with a 50\% increase in the diameter, rotations are reduced by about $15 \%$. This does not mean a better performance for pipes with larger diameters because with increasing the diameter, maximum allowable rotation is reduced (the rotation without leakage and damage). On the other hand, by comparing Table 3 and Table 4, it can be concluded that while the rotation of the critical points in East-West case is less than the rotation of the critical points in North-South case, the stress in these points is larger than the critical points in the perpendicular direction, which is due to the types of connections used in each direction. The stiffness and ultimate strength of Bolted gland connections are larger than those of Tyton connections.

\section{Conclusions}

In general, when seismic waves propagate in the earth's surface, phase differences and frequency contents of an incoming wave are the factors contributing in the failure of buried pipe networks. According to the analysis conducted in the present study, the following results are obtained.

1) In pipes of larger diameters, because of the higher flexural stiffness, less rotation occurs under the same relative displacement imposed from the soil. Considering the rotations of 200 and 300 pipelines, it can be seen that with $50 \%$ increase in the diameter of the pipe, rotations decline by almost $15 \%$. This does not mean that pipes with larger diameters performed better because with increasing diameter, the maximum allowable rotation without leakage and damage is reduced.

2) For modeling boundary conditions, the best scenario, during which the least local stress is caused, is fixed rotations and free translations in all three directions at both ends of a pipeline.

3) Since relative displacement is the cause of damage, in pipes orthogonal to the wave direction no damage occurs, for there is no time lag and successively no relative displacement between different points.

4) As expected, by increasing the velocity and consequently decreasing the phase difference, damages are re- 
duced. By increasing the velocity from 150 to $200 \mathrm{~m} / \mathrm{s}$, stress level in plastic range decreases by $2 \%$, and by increasing the velocity from 200 to $300 \mathrm{~m} / \mathrm{s}$, stress level is decreased by $12 \%$ within the elastic range and the plastic limit.

\section{References}

[1] O’Rourke, M.J. and Liu, X. (1999) Response of Buried Pipelines Subjected to Earthquake Effect. MCEER Report, MCEER-99-MN03|4/29/1999|272 pages, University at Buffalo, Buffalo, NY 14260-4300.

[2] Takada, S., Hassani, N. and Rasti, R. (2003) Artificial Neural Network (ANN) Modeling for Earthquake Damage Detection in Water Distribution System. Proceedings of the 2003 Pacific Conference on Earthquake Engineering [Electronic Resource], 13-15 February 2003, Christchurch, 9 p.

[3] Jayaram, N. and Baker, J.W. (2009) Efficient Sampling and Data Reduction Techniques for Probabilistic Seismic Lifeline Risk Assessment. Earthquake Engineering and Structural Dynamics, 39, 1109-1131.

[4] Toprak, S. and Taskin, F. (2007) Estimation of Earthquake Damage to Buried Pipelines Caused By Ground Shaking. Natural Hazards, 40, 1-24. http://dx.doi.org/10.1007/s11069-006-0002-1

[5] Shi, P., O’Rourke, T.D., Wang, Y. and Fan, K. (2008) Seismic Response of Buried Pipelines to Surface Wave Propagation Effect. Proceeding of the 14th World Conference on Earthquake Engineering, Beijing, 12-17 October 2008, 8 p.

[6] Toprak, S., Taskin, F. and Koc, A.C. (2009) Prediction of Earthquake Damage to Urban Water Distribution Systems: a Case Study for Denizli, Turkey. Bulletin of Engineering Geology and the Environment, 68, 499-510. http://dx.doi.org/10.1007/s10064-009-0230-1

[7] Abdoun, T.H., Ha, D., O’Rourke, M.J., Symans, M.D., O’Rourke, T.D., Palmer, M.C. and Stewart, H.E. (2009) Factors Influencing the Behavior of Buried Pipelines Subjected to Earthquake Faulting. Journal of Soil Dynamics and Earthquake Engineering, 29, 415-427. http://dx.doi.org/10.1016/j.soildyn.2008.04.006

[8] Karamitros, D.K., Bouckovalas, G.D. and Kouretzis, G.P. (2007) Stress Analysis of Buried Pipelines at Strike Slip Fault Crossing. Soil Dynamics and Earthquake Engineering, 27, 200-211. http://dx.doi.org/10.1016/j.soildyn.2006.08.001

[9] J. Kim, O’Connor, S., Nadukuru, S., Lynch, J.P., Michalowski, R., et al. (2010) Behavior of Full Scale Concrete Segmented Pipelines under Permanent Ground Displacements. Health Monitoring of Structural and Biological Systems, 7650, 76500U-1-76500U-11. http://dx.doi.org/10.1117/12.847735

[10] Boorboor, A. (2013) Analysis of Seismic Behavior of Water Distribution Jointed Pipes Network Regarding Nonlinear Behavior of Connections. Ms. Dissertation Submitted to the International Institute of Earthquake Engineering and Seismology, Tehran.

[11] American Society of Civil Engineers (ASCE) (1984) Guidelines for the Seismic Design of Oil and Gas Pipeline Systems, Committee on Gas and Liquid Fuel Lifeline, ASCE, Reston.

[12] Hosseini, M. and Tahamouli, R.M. (2014) A Study on the Effects of Surface Transverse Waves on Buried Steel Pipelines Considering the Nonlinear Behavior of Soil and Pipes. Journal of Pipeline Systems Engineering and Practice. http://dx.doi.org/10.1061/41138(386)103 